\title{
Processus d'institutionnalisation de la démocratie industrielle et crises sociales en France et en Allemagne à la fin des années 1960
}

Institutionalizing Dynamics of industrial Democracy and social Crisis in France and in Germany at the end of the 60's

\section{Olivier Giraud, Michèle Tallard et Catherine Vincent}

\section{OpenEdition \\ Journals}

Édition électronique

URL : http://journals.openedition.org/travailemploi/4651

DOI : 10.4000/travailemploi.4651

ISSN : 1775-416X

Éditeur

DARES - Ministère du Travail

Édition imprimée

Date de publication : 30 juin 2007

Pagination : $39-52$

ISSN : 0224-4365

Référence électronique

Olivier Giraud, Michèle Tallard et Catherine Vincent, « Processus d'institutionnalisation de la

démocratie industrielle et crises sociales en France et en Allemagne à la fin des années 1960 », Travail et Emploi [En ligne], 111 | juillet-septembre 2007, mis en ligne le 15 septembre 2009, consulté le 19 avril 2019. URL : http://journals.openedition.org/travailemploi/4651 ; DOI : 10.4000/

travailemploi.4651 


\title{
Processus d'institutionnalisation de la démocratie industrielle et crises sociales en France et en Allemagne à la fin des années 1960
}

\author{
Olivier Giraud (*) Michèle Tallard ${ }^{* *}$ ) Catherine Vincent(***)
}

\begin{abstract}
Des processus d'institutionnalisation différents ont caractérisé l'évolution des systèmes de démocratie industrielle en France et en Allemagne dans les années 60 et 70 et en particulier des fortes crises sociales des années 1968 à 1969. L'article met en perspective deux modèles contrastées de renouvellement des relations sociales, à partir de rapports différents à la sphère politique de la part des acteurs de la sphère des relations industrielles. Il va à l'encontre de stéréotypes touchant la construction des deux systèmes de relations professionnelles.
\end{abstract}

La comparaison du rôle de l'État dans la sphère des relations industrielles en France et en Allemagne relève bien souvent d'images stéréotypées opposant l'interventionnisme étatique hexagonal à une configuration d'outre-Rhin marquée par l'autonomie des acteurs sociaux et le retrait de l'État. S'interroger sur la pertinence de cette opposition conduit à délimiter un objet de recherche et à analyser, de façon diachronique et comparative, le mode d'élaboration des règles qui touchent à cet objet, c'est-à-dire notamment les rôles respectifs joués par l'État et les acteurs sociaux. La notion de démocratie industrielle, que nous avons choisi de privilégier dans cet article, est à la fois ancienne (WeBB, 1897) et générique; elle ouvre au comparatiste la possibilité d'embrasser dans un questionnement unique des arrangements et des notions qui ont émergé dans des contextes historiques et nationaux qui les ont surdéterminés. En outre elle dessine un point de contact entre «les principes et les procédures de la démocratie politique et la sphère industrielle» (KING, van de VALL, 1978, p. 4) qui permet de mettre l'analyse du rapport au politique des acteurs des relations professionnelles au centre de la comparaison des processus d'évolution de la démocratie industrielle. Ces acteurs ont-ils des stratégies d'investissement du politique qui permettent d'expliquer les formes que prennent dans différents cas nationaux les institutions des relations industrielles? À l'inverse, les

(*) CMB-CNRS, olivier.giraud@ @mb.hu-berlin.de

(**) IRISES-CNRS/université Paris-Dauphine, michele.tallard @ dauphine.fr

(***) IRES Catherine.vincent@ires-fr.org

Cet article s'appuie sur une recherche en cours financée par la DARES (État et acteurs sociaux en France et en Allemagne, dans les décennies 1960 et 1970) à laquelle participe également Michèle Dupré (MODYS-CNRS). formes démocratiques, les modalités d'organisation de la concertation sociale ou les procédures d'institutionnalisation ont-elles un impact sur les formes de la démocratie industrielle entendue ici comme l'organisation des rapports de pouvoir entre travail et capital dans l'entreprise? La démocratie industrielle renvoie aux formes de la représentation des salariés dans l'entreprise, à leurs droits d'information, de protection, aux diverses formes d'association des salariés à la décision dans l'entreprise ou encore aux rapports avec les gestionnaires de l'entreprise. Elle permet de rendre visible la multiplicité des arènes de confrontation entre capital et travail, entre patronat et syndicats, mais aussi entre l'univers du politique et celui des luttes sociales. L'acteur politique ne peut alors être appréhendé au travers d'une entité étatique abstraite. Cette entité doit être décomposée en trois éléments: les partis politiques et la part qu'ils font dans leurs orientations aux propositions syndicales ou patronales; les gouvernements et parlements, et leurs modes de concertation avec les acteurs des relations professionnelles dans l'élaboration des décisions ; les administrations du travail et leur action de préparation et de mobilisation des acteurs dans l'élaboration et la mise en œuvre des réformes.

Un regard historique croisé, embrassant l'aprèsguerre à la décennie 70 , sur les modes d'institutionnalisation de la démocratie industrielle conduit à questionner les spécificités nationales et à mettre l'accent sur les modalités complexes de transferts de notions entre les deux configurations étudiées (DupRÉ et alii, 2006). L'ambition de cet article est de mettre en perspective sur ce même objet de la démocratie industrielle, l'évolution, dans les deux pays, des formes de rapport au politique des acteurs de la sphère des relations industrielles. C'est donc 
cette variable explicative qui sera ici la cheville ouvrière de la comparaison des dynamiques d'institutionnalisation de la démocratie industrielle. Notre démarche se centre sur une variable articulant acteurs et société, considérée comme un analyseur, pour éclairer l'évolution des formes institutionnelles de la démocratie industrielle et elle se place de ce fait dans une logique proche de celle de l'analyse sociétale (Maurice, Sellier, Silvestre, 1982). Cependant, en s'efforçant de saisir et de mettre en rapport les trajectoires de deux configurations nationales au regard d'un enjeu commun, elle s'inscrit dans la perspective de l'histoire croisée (ZIMMERMANN et alii, 1999) et ouvre la possibilité de dépasser le caractère statique de la première approche.

Enfin, cet article est centré sur les décennies 60 et 70 , période clé dans l'évolution des systèmes de démocratie industrielle en France et en Allemagne, et plus particulièrement sur le moment que constituent les fortes crises sociales des années 1968 à 1969 dans les deux pays. Ces deux crises se manifestent à la fois dans le champ du travail - grèves massives - et dans le champ politique. Leur analyse permet ainsi de bien comprendre les articulations entre les deux univers qui nous intéressent ici.

Le rapport au politique se développe en fonction des étapes constitutives du cycle de la réforme et du positionnement politique des acteurs impliqués. Trois étapes principales peuvent être distinguées: celle du débat public, celle de la décision politique et celle de sa mise en œuvre(1). À ces trois étapes correspondent les trois activités essentielles de l'action publique: le discours (au sens de voice), qui renvoie à la capacité à formuler un problème public ; le pouvoir d'institutionnalisation, qui correspond à la capacité à pérenniser des arrangements sociaux dans l'ordre des règles institutionnelles et enfin, la capacité à agir sur le terrain (c'est-à-dire à traiter concrètement le problème public). Plus encore, ces trois activités se déroulent dans des arènes de pouvoir spécifiques et mobilisent des réseaux d'acteurs qui le sont également.

La phase de confrontation entre les discours est une phase au cours de laquelle s'affrontent des représentations et des idées. En la matière, les syndicats, les associations patronales, mais aussi les partis politiques et différentes instances de l'État participent aux échanges. Les enjeux qui s'engagent à ce stade ne sont pas seulement discursifs. Des alliances entre groupes disposant d'une légitimité politique et groupes sociaux (syndicats, autres associations, clubs ou réseaux) peuvent se nouer.

(1) Ces étapes ne constituent pas des séquences qui se succèdent de façon linéaire, mais elles sont souvent intriquées les unes dans les autres.
La phase de l'institutionnalisation est décisive dans la réforme des systèmes de relations industrielles. Elle renvoie à la capacité des acteurs politiques (partis, instances gouvernementales et parlementaires) à fixer des arrangements sociaux en les transformant en des arrangements institutionnels. Les ressources pertinentes sont ici moins directement politiques - capacité à fonder une coalition qui repose sur un soutien populaire ou sur une force de conviction des partenaires - que proprement institutionnelles. Les acteurs gouvernementaux ou les majorités politiques peuvent imposer des normes institutionnelles en se passant de relais sociaux. Cependant, dans le domaine des relations industrielles, le soutien, la négociation, au moins avec des représentants de ces groupes, semblent difficilement contournables. Au-delà cependant des acteurs politiques et des arènes explicites de l'institutionnalisation - le gouvernement, le parlement - un important travail d'institutionnalisation est accompli par les administrations.

La dernière phase est celle de la mise en œuvre; elle renvoie à la mobilisation des acteurs de terrain et à leur volonté de se saisir, d'animer les cadres institutionnels - le plus souvent des instruments d'action publique - définis lors de l'étape précédente.

Dans cet article, nous nous centrerons sur les deux premières phases et nous porterons un regard croisé sur le contenu des débats relatifs à la démocratie industrielle, sur la constitution des coalitions d'acteurs préparant la phase d'institutionnalisation et sur les modes d'institutionnalisation de la démocratie industrielle. En conclusion, nous reviendrons sur la place qu'il convient de donner au rapport au politique des acteurs dans l'éclairage des dynamiques différenciées d'institutionnalisation de la démocratie industrielle.

\section{France et Allemagne: quelles dynamiques discursives?}

Des deux côtés du Rhin, le débat des années 1960 marque la fin de la période de reconstruction. En France, la fin de la guerre d'Algérie libère l'espace politique et une phase importante de modernisation de l'appareil industriel et des relations sociales dans l'entreprise débute. Il s'agit de construire dans les secteurs concurrentiels des entreprises compétitives au niveau international et, dans le champ des relations professionnelles, de légitimer le rôle des organisations syndicales et patronales dans la régulation sociale et d'élargir les pouvoirs des salariés et de leurs représentants dans l'entreprise. Dans ce dernier domaine, ces idées, peu soutenues par les acteurs économiques et sociaux, sont présentes au ministère du Travail tout au long de la période (1958 fin des années 1970). Elles sont défendues soit par des ministres gaullistes de gauche, soit par des centristes ou encore des démo- 
crates chrétiens. Ils essaieront de faire avancer ces objectifs qui seront rarement prioritaires dans une action gouvernementale privilégiant alors les impératifs industriels. Les moments de hausse de la conflictualité (1966-1967), de crise sociale (mai 1968) ou de modernisation de la vie politique (1974) leur ouvrent une fenêtre d'opportunité pour faire passer des réformes allant plus ou moins radicalement dans la voie d'une modernisation sociale.

En Allemagne, la modernisation de l'économie est plus avancée et le pays a retrouvé une place de leader parmi les nations industrielles. L'ordre politique et les rapports sociaux restent cependant marqués par le conformisme et les contraintes liées à la situation internationale du pays. La confrontation avec le passé se focalise au plan économique et social, sur la période de la République de Weimar. La jeune République fédérale d'Allemagne s'entoure de garde-fous censés l'immuniser contre les dérives des années 1920 et 1930. L'équilibre de la monnaie est valorisé; en revanche, le pouvoir des cartels économiques, l'influence des syndicats et du patronat dans la vie économique ou encore le poids de l'État sur l'organisation de l'économie comptent parmi les éléments dont la première version de l'économie sociale de marché cherche à se départir le plus clairement. Les lois imposées au tournant des années 1940 et 1950 par le gouvernement fédéral chrétien-démocrate dans le domaine des relations professionnelles, et notamment l'autonomie tarifaire, garantissent pour l'essentiel une mise à distance entre politique et économique mais reconnaissent le rôle et la capacité du patronat et du syndicat à prendre en charge certaines régulations. Le modèle qui se dessine s'inscrit dans la continuité du répertoire institutionnel allemand construit de la fondation de l'Empire jusqu'à la chute du régime de Weimar.

La confrontation des discours en matière de réorganisation et de rénovation des rapports de pouvoir dans l'entreprise s'engage dans des contextes nationaux dans lesquels ni les clivages politiques ni les enjeux des affrontements ne se recoupent véritablement. En France, pour le pouvoir politique, la modernisation de l'appareil productif ne passe pas par celle des relations de pouvoir dans l'entreprise. L'ambitieux projet gaulliste de participation reste très en retrait par rapport aux aspirations des gaullistes de gauche et de de Gaulle lui-même, en partie en raison de la faiblesse des relais dont dispose cette famille politique dans le monde social et singulièrement dans le monde salarié. Le débat sur l'entreprise s'exprime sous des formes différentes dans de multiples regroupements de la société civile (club, syndicats, partis politiques) et dans des instances de concertation comme le Commissariat général du Plan ou le Conseil économique et social.

En Allemagne, la forte exposition du pays au contexte de la guerre froide pousse les acteurs poli- tiques à la modération idéologique. L'État joue un rôle plus discret qu'en France dans le domaine des relations professionnelles. Pour autant, les revendications syndicales portant sur la socialisation de l'économie comme sur le renforcement des pouvoirs des syndicats dans l'entreprise sont résolument combattues par le gouvernement fédéral chrétiendémocrate et par le camp patronal.

\section{Les années 1960: \\ des positionnements syndicaux contrastés entre les deux pays}

En France comme en Allemagne, les années 1960 sont une décennie d'ouverture et d'élargissement de l'espace démocratique. Cette dynamique emprunte cependant dans les deux pays des voies différentes. En France, les acteurs qui s'activent autour des questions de modernisation des relations sociales dans l'entreprise ne sont jamais dominants dans leurs camps. En dehors d'un réseau d'acteurs où les syndicats prennent de plus en plus d'importance, la question n'est prioritaire ni du côté de l'État, ni bien sûr, de celui du patronat. Mai 1968 représente en France une rupture, «un changement d'échelle» (REYNAUd, 1999, p. 65) dans les visions et les stratégies des acteurs, qui permet le mouvement de réformes sociales de la période 1969-1972. Dans le cas allemand, les positions de départ sont plus arrêtées. La revendication de bouleversement des relations de pouvoir dans l'entreprise est un élément clé du projet de société porté par les syndicats de gauche. Elle suscite une hostilité profonde de la part du patronat, mais aussi de la droite au gouvernement qui cherche dans un premier temps à allumer des contre-feux.

En France, le projet de modernisation sociale s'incarne d'abord dans la volonté de remédier au déclin des comités d'entreprise (CE). En effet, à la Libération, dans la dynamique de la promulgation de l'ordonnance de 1945 et de la loi de 1946, les CE, dominés le plus souvent par la CGT, se multiplient mais, à partir de 1947, la montée des grèves et l'évolution de la stratégie du parti communiste dans la guerre froide changent la donne(2). Face à la CGT qui veut faire de ces institutions des instruments de combat contre le capitalisme, le patronat se raidit et en conséquence, le nombre de CE diminue fortement dans les années cinquante et soixante (LE Crom, 2001).

(2) «Les délégués ouvriers aux comités d'entreprise ne peuvent agir en 1948 comme en 1945 [...]. Avant tout, il faut paralyser l'effort de ceux qui conduisent le pays à la ruine, au chaos, au chômage, à la domination étrangère et à la guerre pour les expansionnistes américains. Les comités d'entreprise peuvent et doivent jouer un rôle capital dans cette action. Ils peuvent et doivent dresser un acte d'accusation contre les responsables de la situation présente et de la misère des masses » (FRACHON, 1948, p. 7) 
Toutefois, une effervescence, souvent en dehors des partis, règne autour de la transformation de la place des salariés et de leurs représentants dans les choix sociétaux et dans le fonctionnement des entreprises. Les débats se cristallisent autour de quelques idées clés: planification démocratique, démocratie dans l'entreprise, participation. Mais suivant les mouvements qui les portent, ces idées ont des contenus diversifiés, cette diversité pouvant exister dans la même organisation. La CFTC amorce au congrès de 1959 un tournant décisif vers sa mutation dans la CFDT par l'adoption à une majorité des trois quarts des mandats d'un rapport préconisant la mise en œuvre de «la planification démocratique»(3), entendue comme plus contraignante que la planification indicative à la française: la reconnaissance de la section syndicale d'entreprise et l'élargissement du rôle des comités d'entreprise s'y combinent à un certain nombre de nationalisations pour assurer le contrôle économique des syndicats; ce contrôle peut éventuellement s'étendre jusqu'aux entreprises, les positions sur ce dernier point étant assez ambiguës (Georgy, 1995)(4). On retrouve également derrière ce vocable les tenants du Plan comme «ardente obligation», au premier rang desquels les gaullistes de gauche alors très présents au ministère du Travail(5), mais également des hauts fonctionnaires du Commissariat général du Plan(6), pour lesquels il s'agit d'associer les représentants du monde du travail à l'élaboration du Plan et à son exécution et, pour cela, de s'appuyer tant sur un meilleur fonctionnement des commissions du Plan que sur un élargissement sous diverses formes des prérogatives des instances représentatives du personnel dans l'entreprise.

(3) G. Declercq, animateur de la commission qui rédigea ce rapport, dira quelques années plus tard à propos du slogan de la planification démocratique, qu'il fut «le pavillon d'un syndicalisme lancé à la recherche d'une identité nouvelle» (cité par GeOrGi, 1995, p. 38)

(4) Pour caractériser l'ambiguïté entre contrôle et participation à la gestion, cet auteur cite un extrait de ce rapport (p. 36): «Les entreprises publiques ou privées auront à déterminer avec les organisations de travailleurs les moyens d'atteindre les objectifs. Ceci nécessite une forme concrète de démocratie qui descendra jusqu'à l'usine et l'atelier par le moyen d'organismes de gestion et de contrôle».

(5) Notamment, à partir de 1962, avec le ministre Gilbert Grandval et son directeur de cabinet Bernard Ducamin lequel quittera le ministère en 1966 pour aller au cabinet de l'Elysée où il appuiera les versions les plus radicales des ordonnances de 1967 sur la participation.

(6) Pour Jacques Delors, alors responsable du service des affaires sociales du Commissariat général du Plan, de la dynamique de consultation engendrée par l'élaboration du Plan se dégage «une sorte de prédiction créatrice qui fait que les conclusions des commissions de modernisation deviennent la charte de l'action des divers partenaires entrepreneurs et syndicats de salariés» (1965, p.154).
Même s'il ne s'agit pas pour la CGT de la première priorité(7), l'extension des droits syndicaux, dans lesquels figurent les droits d'information économique des $\mathrm{CE}$, est présente dans l'accord d'unité d'action CGT-CFDT du 10 janvier 1966 et dans la lettre que ces deux centrales envoient en février 1967 au CNPF pour réclamer l'ouverture de négociation d'un accord interprofessionnel. Refusant tout contact avec la CGT, FO ne se joint pas à ces initiatives; sa position constante est de ne réclamer une extension des prérogatives des $\mathrm{CE}$ que dans la mesure où elles n'empiètent pas sur le pouvoir du syndicat dans l'entreprise.

Côté allemand, les idées qui circulent ne sont pas éloignées dans leur contenu. Le syndicat DGB avait fondé son programme politique en 1949 autour d'un projet de construction d'un ordre socialiste reposant sur la codétermination paritaire (paritätische Mitbestimmung) dans l'entreprise, la collectivisation des industries clés du pays et le développement d'une planification économique étatique forte. Le programme décidé au congrès de Düsseldorf en 1963 est moins radical. La Mitbestimmung et la socialisation de certaines branches de l'économie conservent une place centrale dans le programme syndical. La planification étatique s'est en revanche transformée en une «planification cadre» et en une «orientation des investissements» (Hensche, 1976, pp.689-690). Le recentrage porte surtout, en écho au congrès du SPD de Bad-Godesberg de 1959, sur la reconnaissance du lien positif entre économie de marché et démocratie (HöPNER, 2004). Suite à l'obtention de la codétermination paritaire au sein des conseils de surveillance des grandes entreprises du charbon et de l'acier en 1951, la loi de 1952, constitution de l'entreprise, rétablit les droits d'information des conseils d'entreprise (Betriebsräte), organe de représentation des salariés dans l'entreprise, et n' accorde qu'un tiers des sièges du conseil de surveillance dans les grandes entreprises des autres secteurs de l'économie. En dépit de ces progrès conséquents à une époque où les industries du charbon et de l'acier jouaient un rôle clé, l'échec de l'extension de la parité à toute l'économie et le maintien de la non-reconnaissance du syndicat dans l'entreprise constituent une double déception qui contribue à figer les oppositions et les fronts. À la suite de l'IG-Metall, les syndicats allemands surmontent leur opposition aux Betriebsräte en en prenant le contrôle. Simultanément, au plan politique, ils s'investissent dans une entreprise de mobilisation et de recherche d'alliances. Il s'agit d'imposer la généralisation de la codétermination paritaire au sein des conseils de surveillance des entreprises (THELEN, 1991) mais également de réclamer l'ouverture du

(7) Georgy (1995, p. 315) fait état des débats qui ont présidé à l'accord d'unité d'action dans lesquels, pour la CGT, les revendications de pouvoir d'achat et de conditions de travail devaient précéder celles sur l'extension des droits syndicaux alors que la CFDT mettait cette revendication à la première place. 
capital des entreprises aux salariés. La position syndicale, si elle bénéficie du soutien clair de l'aile gauche de la CDU, des syndicats chrétiens, et du parti social-démocrate, suscite l'opposition farouche de la majorité des gouvernements chrétiens-démocrates de l'époque ainsi que du patronat dans son ensemble.

Alors qu'en Allemagne, le débat sur la réforme des relations de pouvoir dans l'entreprise est largement rendu public, que les clivages sont connus voire dramatisés, en France, les réseaux mobilisés sont plus circonscrits. Ainsi, les propositions contenues dans le livre de François Bloch LaIné Pour une réforme de l'entreprise (1963) résultent largement de débats ayant d'abord eu lieu au sein du club Jean-Moulin dont l'auteur est un proche. Ce club visait à la modernisation de la société par l'approfondissement de la démocratie; il regroupait des hauts fonctionnaires, universitaires, syndicalistes (principalement de la CFTC) et représentants des courants modernistes du patronat (ANDRIEU, 2002). Ce livre préconise de donner plus de vie au comité d'entreprise en distinguant plus précisément information, consultation et contrôle, la participation à la décision ne devant pas dépasser les œuvres sociales. Il met également en exergue la démocratie industrielle «qui contribuera à donner un nouveau visage, une force nouvelle à la démocratie politique»(p.7), recommande la reconnaissance de la section syndicale d'entreprise «parce que la force syndicale est, à juste titre, considérée comme la condition première de la réalité et de l'efficacité de ce pouvoir compensateur que le personnel doit constituer» (p. 83). La section syndicale serait donc dans ce schéma la cellule de base d'un pouvoir syndical. Ces propositions vont être débattues dans des arènes plus larges comme le Plan ou le Conseil économique et social.

C'est, en effet, à cette même nécessité de constituer un pouvoir syndical à partir de la reconnaissance de la section syndicale que conclut le rapport Mathevet (1965), rédigé au début de 1964 par une commission du Conseil économique et social animée par une personnalité active dans la mutation de la CFTC. Constatant la transformation des conditions de production et des conditions de vie et s'appuyant notamment sur des travaux récents d'universitaires et de juristes (8), le rapporteur propose la reconnaissance du syndicat dans l'entreprise afin d'équilibrer et de contrebalancer le pouvoir patronal. Une telle orientation ne pouvait que heurter de front les représentants du monde patronal qui ont toujours

(8) Colloque de l'Institut d'études sociales de l'université de Grenoble sur la reconnaissance de la section syndicale d'entreprise, 24, 25 mai 1963; VII ${ }^{\mathrm{e}}$ colloque juridique organisé à Paris les 28, 29 et 30 juin 1963 sur les droits et les libertés syndicales. refusé cette présence institutionnelle(9). L'avis sera rejeté à une faible majorité(10) mais les positions formulées à l'occasion de ce débat résument bien le climat qui règne, dans les années 1960, autour de la question de la place des syndicats aux différents niveaux de la régulation sociale. L'extension des droits des salariés dans l'entreprise, qu'il s'agisse des pouvoirs du CE ou de la reconnaissance de la section syndicale d'entreprise, cristallise les oppositions. L'élaboration de dispositifs législatifs en la matière sera donc un processus difficile qui n'aboutira réellement qu'à l'occasion de la crise de 1968.

\section{Les réponses étatiques}

Dans les deux pays, l'État est interpellé par les débats qui se développent autour de la question de la rénovation des rapports de pouvoir dans l'entreprise. De part et d'autre du Rhin, les questions de la participation, de l'intéressement des salariés, puis la question de l'articulation entre les régulations produites par les acteurs des relations industrielles et les politiques économiques sont les premières réponses livrées par les gouvernements conservateurs au pouvoir.

Ainsi, en France, il existe un consensus fort sur le rôle des syndicats et du patronat dans les branches professionnelles, mais aussi au sein des commissions du CES ou du Commissariat général du Plan. La planification est alors un «réducteur d'incertitude»; ses responsables se vivent comme des «ingénieurs sociaux» qui inventent des indicateurs et orchestrent des procédures complexes de concertation pour l'élaboration et l'exécution du Plan (Delors, 1965; Delors, 2003). Cette volonté de faire avancer la concertation avec les partenaires sociaux s'exprime également à travers les différentes tentatives de mise en place d'une politique des revenus au début des années 1960. Si la CGT et FO sont plutôt hostiles à cette démarche qui pour la première n'est pas envisageable dans le système capitaliste et pour la seconde nuit à la liberté de négociation, la CFTC en accepte l'idée, mais y pose des conditions en termes de contenu, de déroulement des négociations, d'élargissement des prérogatives des CE et des droits syndicaux dans l'entreprise. Au-delà de la frilosité gouvernementale dans la réponse à ces conditions, le contexte du plan de stabilisation mis en place en septembre 1963 ne favorise pas le succès de ces tentatives.

(9) Les déclarations des représentants du patronat lors du débat sur cet avis sont à cet égard significatives: "Il n'y a pas vraiment de droit syndical, il y a pour les syndicats un droit à être syndiqué, ce qui est différent» (CNPF); «l'entreprise, cellule économique de base, ne peut admettre de corps étranger qui ne se rallieraient pas à la cause commune» (Centre français du patronat chrétien, CFPC) (cité par GEORGI, 1995, p. 73).

(10) Votent pour CGT, CFTC, FO et certains éléments du Centre national des jeunes agriculteurs et de l'UNAF. Votent contre CNPF, CFPC, le reste du CNJA et les autres organisations de l'agriculture. L'abstention de la CGC permet de faire basculer le vote. 
Thème de la première ordonnance signée par le général de Gaulle lors de son retour au pouvoir, la participation est au cœur de la doctrine gaulliste de l'association capital-travail. Mais cette ordonnance du 7 janvier 1959 n'instaure qu'un régime facultatif d'intéressement des travailleursà l'entreprise (11) qui ne connaîtra qu'un faible retentissement en termes de nombre d'accords conclus. Cette thématique ne sera reprise, sous la pression de de Gaulle lui-même et par le truchement des ministres et parlementaires gaullistes de gauche, qu' au milieu des années 1960, pour pousser le gouvernement Pompidou à préparer les ordonnances de 1967 instaurant un régime limité mais obligatoire. Sans doute, les préférences du gouvernement Pompidou pour l'industrialisation expliquent-elles cette «course de lenteur» (GuYot, 2001) mais le peu d'intérêt manifesté par les syndicats pour ces procédures et les freins mis par le patronat n'ont pas favorisé le développement des formules de participation.

En Allemagne, les revendications de la gauche, notamment syndicale, en faveur d'une redistribution des cartes du pouvoir économique crée un clivage au sein même du parti chrétien-démocrate. Le courant ouvrier de la CDU adopte une politique d'ouverture vis-à-vis de la gauche modérée et crée, dès le début des années 1960, les conditions d'une coalition générale mais aussi les conditions à la réalisation d'alliances limitées à des objets précis. Le projet de socialisation partielle des moyens de production attaché à la revendication de codétermination suscite, quant à lui, l'attention de la gauche du parti chrétien-démocrate, mais aussi de quelques services ministériels. Dès la fin des années 1950, le gouvernement Adenauer s'est saisi de l'intégration des classes moyennes - le gouvernement fédéral redoute la paupérisation des indépendants-à travers l'action d'un ministère fédéral du Travail investi de nouvelles fonctions. Adenauer lui-même, suivi par des hauts fonctionnaires du ministère comme Günther Schelp, comprend ce concept de classes moyennes (Mittelstand)(12) de façon extensive et développe une réflexion large sur la réforme sociale. Au tournant des années 1950 et 1960, ces cercles se saisissent de la revendication syndicale portant sur l'ouverture du capital des entreprises à leurs salariés, dans le but de promouvoir un capitalisme ouvrier qui aurait une fonction de stabilisation politique et une fonction de consolidation de la situation économique des ouvriers (BOLDORF, 2007). Les projets de lois préparés au sein du ministère fédéral du Travail prévoient un important pouvoir d'information des salariés leur permettant de juger de l'im-

(11) Quatre voies sont possibles: participation collective aux résultats, participation au capital ou à une opération d'autofinancement, participation à l'accroissement de productivité.

(12) Le concept de Mittelstand intègre en allemand à la fois les classes moyennes, le monde des petites entreprises ainsi que, plus largement, les travailleurs indépendants. portance de l'intéressement accordé. Ces projets avortent devant les réticences fortes d'une partie de la CDU, des libéraux, du patronat de l'artisanat et des PME mais aussi de l'opinion publique conservatrice. La publicisation précoce des intentions du gouvernement fédéral a en l'occurrence desservi la stratégie d'Adenauer et du ministère du Travail. La focalisation des débats sur l'identité capitaliste de l'Allemagne constitue au début des années 1960 un clivage trop important pour que puisse être enregistrée une avancée institutionnelle, aussi bien dans le dossier de la répartition du pouvoir dans l'entreprise que dans celui portant sur le régime de propriété de ces mêmes entreprises.

En Allemagne, comme en France avec le Plan, le gouvernement de grande coalition(13) se lance dans la concertation sociale, la konzertierte Aktion. L'action concertée vise à informer les organisations salariales et patronales sur les perspectives de la croissance et des investissements publics et privés dans le pays et les invite à évaluer leurs politiques des revenus en fonction de ces perspectives. Â la fin de l'année 1967, le ministre fédéral de l'Économie, le social-démocrate Karl Schiller, accepte d'adjoindre aux discussions conduites dans le cadre de l'action concertée de nombreux «problèmes de société », de fait très liés à la question du revenu ouvrier: l'intéressement aux bénéfices de l'entreprise ou la constitution d'un patrimoine ouvrier font l'objet de ces discussions.

Les premières réponses concrètes de l'État aux revendications de démocratisation de la vie de l'entreprise restent partielles, que ce soit sur les questions de l'intéressement ou des droits des instances représentatives du personnel. Malgré les pratiques de concertation sociale, ces réponses, apportées par les gouvernements conservateurs français et allemands, ne sont pas considérées comme des compromis acceptables par les syndicats.

\section{Les crises de la fin des années 1960: ruptures et continuités dans les rapports au politique des acteurs}

En France, la crise de 1968 met à jour les dysfonctionnements du système de relations professionnelles et remet en cause les stratégies des acteurs: «Révisant la liste des revendications et définissant à nouveau les priorités, elle bouscule les programmes établis » et «réintroduit au devant de la scène l'arbitrage gouvernemental qui s'exerçait avec discrétion ou avec effacement.» (REYNAUD, 1999, p. 69).

(13) La coalition réunit le SPD et la CDU/CSU. Elle exclut de fait les libéraux (FPD) qui forment le seul parti significatif d'opposition politique parlementaire. 
L'action gouvernementale va se manifester dans le social-delorisme, tentative de réformes sociales concertées avec les acteurs sociaux, menée après l'élection de Georges Pompidou par son nouveau Premier ministre Jacques Chaban-Delmas dans la période 1969-1972. Cette crise n'a pas d'équivalent en Allemagne, mais les grèves sauvages de septembre 1969 et, de manière plus générale, la hausse des conflits du travail au tournant des années 1960 et 1970 va jouer le rôle de révélateur de la contestation de la large coalition politique (19661969) et de la Konzertiete Aktion, portant avant tout sur l'orientation des normes et formes de croissance, et auxquelles participent les organisations patronales et syndicales. La volonté d'en finir avec les pratiques de modération salariale et la focalisation au centre de l'espace politique, caractéristiques de la grande coalition, sont peu prises en compte par le syndicat. Ces facteurs expliquent l'ampleur des grèves sauvages de septembre 1969.

Dans les deux pays, la sortie de crise est marquée par une réévaluation de l'intervention de l'État dans les relations sociales mais, surtout, par le repositionnement des acteurs patronaux et syndicaux dans le champ politique. La vision que les syndicats ont développée de leur inscription dans une perspective de changement politique est renouvelée au travers d'une nouvelle articulation entre l'arène des relations industrielles et l'arène partisane: soutien au programme commun entre le PS et le PCF en France, alliance solide entre le DGB et le SPD en Allemagne.

En France, le programme gouvernemental de la «nouvelle société» se donne comme objectif de transformer les relations professionnelles, alors marquées par la logique du conflit, au travers du développement de la négociation collective interprofessionnelle et dans les entreprises nationalisées au travers de contrats de progrès (14) ouvrant à une programmation concertée des rémunérations à moyen terme (JOBERT, 2000). Ce projet est partagé par des hauts fonctionnaires qui investissent l'appareil d'État et des intellectuels qui prônent un changement profond des relations sociales (15). Leur arrivée au sein du gouvernement va un temps favoriser cette tentative réformiste. Jacques Delors, conseiller social de Chaban-Delmas de 1969 à 1972, est sans doute le plus important représentant de cette voie. Pour rénover la société bloquée(16) et relancer la politique contractuelle, il faut mettre

(14) Il s'agit d'accords pluriannuels (trois ans) prévoyant des formules d'augmentation des salaires liées à des indicateurs économiques nationaux et à la productivité dans les entreprises publiques. Ces contrats prévoyaient initialement une clause de paix sociale qui sera rapidement abandonnée devant les réticences syndicales.

(15) Au sein du club Jean-Moulin ou de celui fondé par Jacques Delors, citoyens 60, ces positions sont anciennes.

(16) Du titre de l'ouvrage de Michel Crozier qui paraît en 1970 . à l'agenda des réformes dans des domaines où, audelà des divergences quant aux projets de société, des positions communes sont possibles entre acteurs. Les compromis seront alors négociés entre patronat et syndicats avant toute traduction législative (Verdier, Langlois, 1972). Les organisations syndicales s'engagent dans cette dynamique sur les procédures de négociation collective, sur l'amélioration des conditions de travail, l'emploi et la formation professionnelle.

En Allemagne, pendant toute la durée de la grande coalition, entre 1966 et 1969, et en dépit des pressions renforcées exercées par les syndicats, ces derniers n'obtiennent pas d'avancée substantielle. En revanche, syndicats et SPD concluent un accord qui porte sur la réalisation du projet syndical, une fois que la gauche sera arrivée seule au pouvoir ou dans une situation de partenaire majeur de la coalition.

Parallèlement, les projets politiques des syndicats évoluent de façon divergente. En France, la CGT et la CFDT élaborent des projets de société incluant de nouvelles formes d'organisation du pouvoir des salariés dans les entreprises.

La stratégie de la CGT après 1968 se comprend en référence à l'impact du mythe de 1936 sur cette organisation (MOURIAUX, 1982): la prise du pouvoir politique par des partis de gauche et la mobilisation sociale animée par une organisation syndicale puissante, la CGT, doivent aller de pair, sans quoi le mouvement ouvrier va à l'échec. C'est ainsi qu' après avoir participé au constat de Grenelle dans la dynamique des conflits de mai 1968, la CGT encourage au repli du mouvement social sur la base du constat de l'absence d'une majorité politique à gauche. Mais le contenu des grèves, la remise en cause des rapports de travail dans l'entreprise amplifiée dans les grèves du début des années 1970, et la place nouvelle faite par ce mouvement social aux jeunes et aux femmes auront également une influence à l'intérieur de la CGT. Ils servent alors de référence à des courants eurocommunistes prônant une plus grande ouverture du syndicat et l'abandon de «l'esprit de bastion» (Moynot, 1982). Suite à la signature du programme commun en juin 1972, la CGT fait de l'accession au pouvoir politique des partis signataires la condition indispensable au changement social. Cette vision conduit à encourager l'unité d'action - l'accord sur un programme d'action signé avec la CFDT en 1970 puis renouvelé en juin 1974 se place dans cette logique - et à subordonner les luttes au changement politique. Toutefois, face aux restructurations industrielles et à l'extension des licenciements, certaines sections syndicales CGT proposent des solutions industrielles favorables aux salariés mais inscrites dans la logique du système capitaliste (17).

(17) Ce mouvement est initié par le conflit Rateau en 1972 
En complément de la planification démocratique, c'est autour de la notion d'autogestion, c'est-àdire la gestion de l'entreprise par ses salariés, que la CFDT du début des années 1970 se construit une identité la démarquant de ses rivales. Celle-ci est fondée sur une articulation originale entre les revendications de court terme et un projet autogestionnaire de changement social. La définition cédétiste de l'autogestion puise dans le fonds commun de la CFTC qui se caractérise par «une aspiration constante à des responsabilités élargies pour tous les travailleurs et dont le terrain d'élection est l'entreprise, perçue comme la cellule de base de l'économie» (GeORGI, 1994, p. 767). À la fin des années 1960, des liens existent surtout entre la CFDT et le PSU, mais les évènements de mai-juin 1968 accélèrent une radicalisation déjà à l'œuvre qui s'accompagne d'une plus nette indépendance. En s'appuyant sur la dynamique du mouvement de grèves, la CFDT élabore un véritable projet politique. Les propositions visent à renforcer la responsabilité et le pouvoir des travailleurs par l'autogestion, le contrôle, la participation à la gestion ou encore la cogestion. L'entreprise est appréhendée dans son lien avec la planification et l'objectif est d'explorer ce que la CFDT veut changer pour démocratiser l'entreprise. Jusqu'à la fin des années 1970, la stratégie de la CFDT sera de développer et de s'appuyer sur les mobilisations ouvrières, comme celle emblématique de Lip, afin de préparer les conditions d'un changement social. Les échecs des grèves d'entreprise et la montée en crédibilité d'une alternative politique autour de l'Union de la gauche entraînent une réorientation vers une relation plus classique où le syndicat espère faire prendre en compte les revendications syndicales.

En Allemagne, le projet politique des syndicats consiste à inscrire la codétermination dans une gestion programmée et intégrée de la société. Un conseil économique et social, regroupant État, partenaires sociaux et société civile, prendrait en charge les politiques sociales mais aussi la politique des revenus, articulées à la gestion de la politique macroéconomique de l'État, en coordination avec les orientations et les investissements consentis dans et par les entreprises privées (KLÖNNE, REESE, 1990, p. 265). En dehors de la mouvance sociale de la CDU, cette version remaniée du projet syndical ne suscite aucun enthousiasme à droite. En revanche, l'essentiel du parti social-démocrate, à l'exception notable du ministre fédéral de l'Économie, soutient l'initiative syndicale pour l'extension de la Paritätische Mitbestimmung.

Sans doute, les différences de configuration des liens avec les partis politiques dans les deux pays éclairent-elles cette différenciation des stratégies syndicales. En revanche, les stratégies patronales sont proches.
En France, mai 1968 provoque également un véritable aggiornamento (MAGNADAS, 1985) dans une organisation patronale attachée à l'autonomie de ses fédérations. Le CNPF refuse, pendant toutes les années 1960, l'institutionnalisation de la présence syndicale dans l'entreprise et la négociation interprofessionnelle. Il ne consent à ce dernier niveau qu'à ce qu'il qualifie dans ses écrits internes «de conversations paritaires » (VINCENT, 1997). Les prémisses d'une évolution apparaissent à partir de 1965 avec l'édiction d'une charte, acte qui permet de «poser le patronat français en acteur capable de proposer une doctrine et d'affirmer des valeurs» (SEllier, 1984, p. 63), l'élection d'un président considéré comme un réformateur et la signature d'un accord sur l'indemnisation du chômage partiel en février 1968. L'intensité du mouvement social de 1968, qui contraint le CNPF à accepter la reconnaissance de la section syndicale, pèsera d'un poids décisif dans le processus aboutissant à un changement de statut de l'organisation patronale. Le président, s'appuyant sur les grands groupes, exploite les tensions internes pour imposer en 1969 une réforme des statuts qui renforce les pouvoirs de l'instance nationale par rapport aux fédérations et en fait un organe d'action et non plus seulement de représentation et de coordination. La confédération a désormais le pouvoir de négocier avec les organisations syndicales, sauf sur les salaires. Dans l'entreprise, les réticences se maintiennent comme le confirment les appels répétés des pouvoirs publics au cours de la décennie 1970 à la reconnaissance pleine et entière du fait syndical.

En Allemagne, les premières consultations tripartites lancées par le gouvernement fédéral en 1963 avaient marqué la fin de la période la plus conservatrice du pouvoir fédéral après-guerre, l'arrivée d'une génération d'experts au sein de l'administration fédérale et l'ouverture de pratiques de pilotage macro-économique (NüTZENADEL, 2005). Elle a également pour conséquence de renforcer la représentation des intérêts patronaux d'employeurs, habituellement ouverts à la négociation sociale (le BDA), face aux intérêts patronaux, plus conservateurs (le BDI)(18), mais aussi de conforter la position des syndicats comme interlocuteurs légitimes (SCHROEDER, 2000, p. 522). Cette implication conduit toutefois également les syndicats à une modération salariale vite contestée par la base. La constitution d'un bloc alliant droite et gauche au sommet du pouvoir politique renforce l'impression d'une absence d'opposition dans le pays. Le salariat s'invite sur la scène politique en se passant de la

(18) En Allemagne, il existe deux filières de représentation des intérêts patronaux: selon une logique avant tout économique - BDI - et selon une logique d'employeurs - BDA. La seconde filière est traditionnellement beaucoup plus proche des revendications syndicales, notamment parce qu'elle a intérêt à un renforcement des régulations de type conventionnel. 
médiation syndicale au moyen des dures grèves sauvages de 1969. Le syndicat est alors forcé de comprendre qu'il lui faut obtenir des résultats probants sur le terrain des droits salariés dans l'entreprise s'il veut reprendre l'initiative. La pression placée sur la revendication en faveur de la codétermination se renforce de nouveau. En 1965, le syndicat avait adressé la proposition de s'adjoindre l'expertise d'une commission officielle. Le gouvernement fédéral se saisit en 1967 de cette proposition. Il confie à un universitaire conservateur, Kurt Biedenkopf, le soin de réunir une commission chargée de faire une évaluation des acquis et des échecs de la loi de 1951 dans les secteurs du charbon et de l'acier et d'adresser, sur la base de ces constats, des recommandations pour le reste de l'économie. Le rapport Biedenkopf, publié en 1970, suscite de vives polémiques. En dépit d'un bilan empirique largement positif de l'expérience de la Montanmitbestimmung, il rejette son extension à l'ensemble de l'économie, invoquant la remise en cause du droit de propriété. Le débat qui s'ensuit se déroule pour l'essentiel entre partis politiques.

Un débat parallèle se développe au parlement. Le DGB dépose une première proposition de loi en 1968, suivi par le SPD la même année. Cinquante projets différents sont discutés entre 1968 et 1973, poussant l'ensemble des partis politiques à définir une position. Chez les chrétiens-démocrates et les libéraux, les débats internes sont très difficiles, seuls les sociaux-démocrates, qui collent à la position des syndicats, ne connaissent pas de véritable déchirement sur cet enjeu. Les associations patronales reprennent leurs mobilisations face aux projets de réforme qui circulent. Les syndicats mobilisent à leur tour l'opinion et menacent directement le SPD: le syndicat donnera des consignes de vote en fonction de l'engagement des partis politiques à propos de l'enjeu de la codétermination dans l'entreprise.

Le travail de construction d'un compromis politique se poursuit dans un espace strictement limité à la coalition gouvernementale sociale-libérale formée en 1969. Alors que le ministère de l'Économie revient aux Libéraux, les oppositions entre les deux membres de la coalition sont vives sur la codétermination: il faudra cinq ans de coordination interne pour aboutir à un compromis qui ne réalisera pas le projet de parité intégrale annoncé dans le discours de politique générale du gouvernement fédéral de janvier 1973.

Dans les deux pays, on voit se profiler des coalitions d'acteurs syndicaux et d'acteurs politiques mais les différenciations persistantes des liens entre la sphère des relations industrielles et la sphère du politique expliquent les dynamiques différenciées d'institutionnalisation.

\section{Des dynamiques}

d'institutionnalisation fortement contrastées: institutionnalisation partielle vs passage en force

\begin{abstract}
À la fin de la période, l'institutionnalisation des rapports de pouvoir dans l'entreprise a connu des avancées importantes en Allemagne (loi de 1972 et de 1976), alors qu'en France, après les avancées partielles des lois de 1966 et de 1968, les vigoureux débats de la décennie 1970 sur la réforme de l'entreprise (rapport Sudreau, cf. DupRÉ et alii, 2006)], ne trouvent pas de débouchés institutionnels avant les lois Auroux de 1982.
\end{abstract}

En Allemagne, le processus d'institutionnalisation s'appuie autant sur la victoire électorale du SPD en 1969 que sur la centralité de ce thème dans les stratégies syndicales. En France, au contraire, malgré l'ampleur des évolutions des conditions de travail négociées au niveau macrosocial, le domaine de l'entreprise où un compromis entre acteurs paraît impossible est évité. D'une part, la majorité politique n'est pas modifiée par l'ébranlement de mai 1968; d'autre part, les syndicats n'ont pas de vision commune sur cet enjeu et sont en attente du changement politique.

En France, la difficulté d'aboutir à un compromis sur le pouvoir dans l'entreprise existe déjà dès le début des années 1960. Une issue institutionnelle n'est trouvée qu'en 1966 et elle se limite à élargir le rôle des comités d'entreprise et à renforcer la place des syndicats en tant que tels en leur sein. Cette loi a connu de nombreuses vicissitudes entre 1962 où elle est mise au programme de la nouvelle législature par le ministre du Travail, Gilbert Grandval, dans un objectif ambitieux d'élaboration d'un programme social visant à l'organisation des relations professionnelles dans l'entreprise (19) et l'adoption quatre ans plus tard d'un projet de loi a minima(20), limitant l'extension du rôle du CE au domaine de l'évolution de l'emploi et de l'adaptation professionnelle et proposant une protection juridique et un crédit d'heures pour le représentant syndical au CE dans les grandes entreprises. Dans l'intervalle, ce projet s'est heurté à la prudence du Premier ministre soucieux, dans une période où la priorité est à l'industrialisation, de ne pas mécontenter le patronat, hostile tant à l'intervention économique des CE qu'à l'institutionnalisation de syndicats dans l'en-

(19) Il s'agit d'assurer par «une meilleure participation des travailleurs aux problèmes économiques et financiers de l'entreprise» un cadre propice à la «planification démocratique» et aboutir à «un syndicalisme fort, mieux au fait des réalités économiques » (CAC 890293, article 6).

(20) Selon l'expression employée par le directeur de cabinet du ministre du Travail durant l'élaboration de ce projet (CAC 890293, article 6). 
treprise. Toutefois, même limitée, une loi finira par aboutir. Alors qu'apparaissent de premières difficultés d'emploi, on peut faire l'hypothèse que la forte conflictualité, initiée par la grève des mineurs de 1963 et qui s'intensifie après 1965(21), ainsi que l'accord d'unité d'action signé début 1966 entre la CGT et la CFDT qui mettait l'extension des droits syndicaux parmi ses priorités, pousse le gouvernement à finalement conclure sur ce qui peut lui apparaître comme un compromis susceptible de satisfaire en partie le patronat - qui obtient que la section syndicale d'entreprise (SSE) ne soit pas formellement reconnue et que l'extension des droits des CE soit limitée - et les syndicats - qui pourront tirer avantage des nouveaux droits du représentant syndical et des CE dans une conjoncture d'emploi qui commence à se tendre.

Durant ce lent processus législatif, dans un certain nombre de grandes entreprises, des accords instituant la reconnaissance de la section syndicale sont signés. Ainsi, comme l'affirmait déjà le rapport Mathevet «l'institution est d'ores et déjà une réalité juridique», pourtant il faudra attendre le mouvement de mai 1968 pour que le patronat accepte la figure du délégué syndical. Lors des négociations de Grenelle, cette reconnaissance est actée et le gouvernement est chargé d'élaborer une loi portant sur cinq points: la garantie des libertés syndicales, la protection des délégués, les prérogatives des syndicats, leurs moyens d'expression et leurs moyens matériels d'existence. L'équilibre entre les positions des uns et des autres est si fragile que la trame du futur projet de loi fait l'objet d'une annexe détaillée du constat, alors que les autres domaines sont renvoyés à la négociation. La loi sera finalement votée en décembre 1968.

Les tentatives de développer des négociations interprofessionnelles entreprises dans la deuxième moitié des années 1960, tant par le gouvernement (22) que par les organisations syndicales, avaient largement échoué(23). La dynamique engendrée par le constat de Grenelle et les orientations volontaristes en matière de négociations sociales portées par le social-delorisme vont conduire à une série d'accords suivis de lois qui, s'ils ne portent pas directement sur l'entreprise et les relations de pouvoir en son sein, ont des conséquences sur les relations sociales à ce niveau. D'une part, ces accords accroissent le rôle des CE: consultation sur les projets de licenciement (ANI 1969), avis sur le plan de formation (ANI 1970). D'autre part, précédée d'une intense activité

(21) Statistiques des grèves hors fonction publique en millions de journées non travaillées: $1964: 2,5 ; 1965: 1,0 ; 1966: 2,5$; 1967: 4,2 (source ministère du Travail).

(22) Lettre de cadrage du Premier ministre aux organisations syndicales et patronales pour les inciter à négocier sur cinq thèmes touchant à l'emploi.

(23) Seule l'indemnisation du chômage partiel donnera lieu à un accord (28 février 1968). de consultation au sein de la commission supérieure des conventions collectives, la loi de 1971, prenant acte de l'émergence d'accords d'entreprises dans les décennies 50 et 60 dans les grandes entreprises et de la reconnaissance de la section syndicale et des délégués syndicaux, place l'entreprise comme un niveau à part entière de négociation (BORENFREUND, 1997). Elle érige les accords d'établissement au rang de véritables conventions collectives et pose l'indifférenciation des niveaux de négociation dont la coordination est assurée par le principe de faveur(24) (RotsChILD-Souriac, 1993). Mais cette faculté de déployer la négociation collective au niveau de l'entreprise sera peu utilisée par les organisations syndicales et la négociation collective d'entreprise ne connaîtra un véritable essor qu'au milieu des années 1980, après les lois Auroux de 1982.

En Allemagne, la phase de confrontation des discours ne débouche pas sur la constitution d'un compromis véritable. L'intervention médiatrice des partis, et notamment de la frange sociale de la CDU, permet cependant une amorce de rapprochement visà-vis d'une position syndicale elle aussi recentrée. La Mitbestimmung n'est plus à la fin des années 1960 prioritairement un instrument qui prépare la socialisation de l'économie, mais elle est une façon d'avancer dans la réalisation du projet de société intégrée porté par le syndicat. La codétermination représente également une avancée des droits des salariés qui permet au syndicat de rattraper l'échec de 1952 et de manifester sa combativité alors qu'il est souvent prêt à jouer le jeu de la modération sur le plan salarial.

Les rapports entre le politique et l'univers des relations professionnelles sont souvent articulés en Allemagne par le jeu de doubles appartenances entre partis et centrales syndicales ou patronales: députés au Bundestag ou ministres cumulent facilement l'étiquette CDU ou SPD et DGB ou syndicat chrétien. Le politique remplit alors une importante fonction d'initiative et institutionnalise des compromis qui ne bénéficient pas de soutiens majoritaires dans l'arène des relations industrielles. Dans le domaine de la démocratie industrielle cependant, la grande coalition qui a pu régler des conflits durs entre la droite et la gauche - le conflit fédéraliste, le blocage dans le domaine de la formation professionnelle (OFFE, 1975) - ne peut trouver une issue satisfaisante malgré les concertations institutionnelles ouvertes dans cette période.

La transformation des rapports de pouvoir dans les entreprises se concrétise avec l'arrivée en 1969 au pouvoir de la coalition SPD-FDP. En premier lieu, la loi organisant la «constitution» de l'entreprise, Betriebsverfassungsgesetz, est remise

(24) Les garanties négociées dans les accords d'entreprises ne peuvent être que plus favorables aux salariés que celles existantes au niveau des conventions collectives de branches lesquelles sont plus favorables que la loi. 
sur l'ouvrage. Enjeu d'une alliance forte entre syndicat et SPD, cette loi fait partie du programme du gouvernement fédéral et chacun la considère comme acquise (Lompe, 1990). Cette loi représente cependant une avancée importante puisqu'elle augmente de beaucoup les droits du Betriebsrat en matière sociale, de gestion du personnel ou encore de contrôle des conditions de travail et clarifie les relations entre Betriebsrat et syndicat. Enfin, les protections juridiques dont bénéficient les individus dans l'entreprise sont renforcées (FrERICH, FrEY, 1993).

En second lieu, la loi fédérale de 1976 sur la Mitbestimmung marque pour les syndicats l'horizon ultime de la revendication en matière de partage de pouvoir. Ces derniers ne se satisfont pas d'une loi qui renforce leurs droits dans quelques domaines, mais exigent bien la parité dans le contrôle de l'entreprise en général. Après que les différentes propositions développées depuis la fin des années 1960 et qui émanaient du camp syndical, patronal ou des différents partis n'aient pas réussi à créer un consensus, la négociation s'est focalisée sur le sommet du pouvoir, entre les partenaires de la coalition gouvernementale. Contrairement au projet social-démocrate initial garantissant la parité intégrale entre salariés et employeurs, le modèle retenu accorde une voie prépondérante au président du conseil de surveillance en cas de blocage persistant. De plus, à la différence de la loi réservée au secteur du charbon et de l'acier de 1951, l'Arbeitsdirektor n'est plus nommé par la partie salariée mais en accord avec elle. La décision du tribunal constitutionnel fédéral accordant aux cadres supérieurs une représentation spécifique a encore renforcé ce trouble dans la parité entre salariés et employeurs.

Le compromis disputé et réduit du point de vue des forces politiques impliquées est contraire à la tradition allemande de mobilisation du consentement (Hall, 1997). Peu cohérente dans son esprit et faible politiquement, la loi de 1976 suscite de très vives oppositions politiques, mais aussi dans la sphère des relations industrielles. Les syndicats se déclarent trahis car leur conception de la parité n'est pas atteinte et quittent de façon démonstrative la Konzertierte Aktion, vidée de son efficacité depuis des années déjà. Le camp patronal se sent dépossédé de son pouvoir dans l'entreprise et se lance dans une campagne acharnée pour obtenir l'annulation de la loi devant le tribunal constitutionnel fédéral.

$\mathrm{Au}$ final, l'institutionnalisation douloureuse de la loi de 1976 contraste avec l'ampleur et les effets positifs de la loi de 1972 sur la constitution d'entreprise, effets positifs largement soulignés par la littérature sur les relations industrielles (STREECK, 1985).

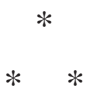

À la fin des années 1960, la similarité relative des situations de crise sociale mais aussi politique, en France comme en Allemagne, renvoie de part et d'autre à un fort besoin de renouvellement des débats, des rapports de force et des rapports de pouvoir dans la société. Alors qu'en Allemagne, c'est autour de la démocratie industrielle que vont se recomposer les compromis sociaux, en France, il n'y a pas de consensus entre les acteurs des relations professionnelles pour se mobiliser sur cet objectif. Au-delà de cette opposition, analysée dans cet article, la comparaison des dynamiques d'institutionnalisation de la démocratie industrielle dans les deux pays nous conduit à privilégier trois dimensions permettant de mettre en perspective les configurations nationales et dégageant un rapport au politique qui structure des dynamiques d'institutionnalisation décalées dans le temps et portées par des ministères du Travail actifs dans les deux pays.

L'analyse de la dynamique des relations sociales dans la décennie 1970 renouvelle également la vision classique des liens syndicats/politique dominante dans les deux modèles de syndicalisme. Revisitée à l'aune de l'enjeu de la démocratie industrielle, la différenciation entre ces modèles ne réside pas tant dans l'opposition entre un syndicalisme réformiste et un syndicalisme révolutionnaire, mais dans la place donnée au politique dans l'articulation entre les revendications immédiates et un projet de changement social. En Allemagne, on observe un glissement par rapport au modèle social-démocrate canonique. Les syndicats ont fait de la prise en compte de la Mitbestimmung dans son programme politique la condition de leur soutien électoral au SPD. La pratique de la codétermination, en ce qu'elle accroît les capacités gestionnaires des représentants des salariés, peut constituer une étape dans le processus de transformation des rapports sociaux, en ce sens elle articule le court et le long terme.

En France, les liens syndicats-partis politiques se complexifient dans la période: au suivisme des années 1950 succède à la CGT une vision plus autonome de l'action syndicale; s'affranchissant du catholicisme social, la CFDT développe un projet politique original axé sur l'autogestion. Pour autant, les deux organisations syndicales subordonnent au changement politique la réalisation d'une démocratisation de l'entreprise. Pour la première, la prise du pouvoir par une coalition dominée par le parti ouvrier permet seule de transformer la société capitaliste, le syndicat alimentant par les propositions et la mobilisation cette transformation. Pour la seconde, si le changement politique est indispensable, il n'est pas suffisant. C'est au travers des luttes et du contrôle des entreprises que l'on peut préfigurer la société future. 
Les débats sur la transformation de la démocratie industrielle font l'objet d'un double décalage entre la France et l'Allemagne. Il s'agit en premier lieu d'un décalage dans la construction historique de l'enjeu. En France, le renforcement du pouvoir des salariés dans l'entreprise ne correspond pas à une revendication traditionnelle du mouvement syndical, mais est majoritairement conçu par des cercles modernisateurs modérés comme un moyen d'agir sur la conflictualité sociale et d'obtenir une amélioration de la condition des salariés par des moyens pragmatiques. En Allemagne, la revendication autour du pouvoir dans les entreprises fait partie du répertoire du monde ouvrier depuis des décennies. D'abord articulée à un projet de socialisation des moyens de production, cette revendication se décline à partir du milieu des années 1960 dans un programme politique plus modéré qui permet de gagner le soutien de la mouvance sociale du parti chrétien-démocrate.

Le second décalage est un décalage temporel. L'endogénéité à la sphère des relations industrielles de la revendication de Mitbestimmung explique qu'en Allemagne le débat s'engage plus tôt qu'en France. Une partie importante du travail politique de recherche d'un consensus s'élabore dès les années 1960, dans une période de conflictualité sociale forte. En France en revanche, le débat est avant tout porté par des acteurs politiques et il n'entre dans une véritable phase d'institutionnalisation que dans le courant des années 1970.
Dans les deux pays cependant, le travail de construction du compromis implique des coopérations étroites entre la sphère des relations industrielles, les réseaux politiques et l'État. En la matière, même si les configurations sociétales sont contrastées, de nombreuses similarités doivent être remarquées. En premier lieu, il faut insister sur la position de pivot remplie par le ministère du Travail. Dans des gouvernements de droite, ce portefeuille est facilement confié à des personnalités représentant les sensibilités, minoritaires, d'ouverture en direction du monde ouvrier - gaullistes «de gauche» ou tendance sociale de la CDU. Dans les deux pays, les ministères jouent un rôle actif en mettant à disposition leurs moyens. Côté allemand, l'expertise juridique des départements ministériels qui accompagnent le développement de l'activité conventionnelle est mise à disposition. Côté français, l'activité du ministère reste importante au moment de la mise en œuvre des dispositifs arrêtés. Les circulaires d'application et le travail administratif dans son ensemble permettent d'influencer la mise en œuvre dans un sens ou l'autre de façon importante. Dans les deux pays, des réseaux sont alimentés de projets, propositions ou de données ou autres éléments de connaissance dans le but de faire avancer le dossier. Enfin, la dernière similarité est sans doute la plus décisive. Dans les deux pays, en dépit de différences importantes dans les systèmes de concertation, l'institutionnalisation ne progresse qu'à la condition d'une prise de pouvoir dans l'arène politique. 


\section{Bibliographie}

Andrieu C. (2002), Pour l'amour de la République, le club Jean-Moulin, 1958-1970, Paris, Fayard.

Bloch-Lainé F. (1963), Pour une réforme de l'entreprise, Paris, PUF.

Boldorf M. (2007), "Sozialpolitische Denk - und Handlungsfelder", in Boldorfn M., M. Ruck M. (sous la direction de), Handbuch der Geschichte der Sozialpolitik in Deutschland nach 1945, Bd. 4: Bundesrepublik Deutschland 1958-1966. Sozialpolitik im Zeichen des erreichten Wohlstandes, Baden-Baden, Nomos.

BorenFreund G. (1997), «Les syndicats forcent les portes de l'entreprise: la loi du 27 décembre 1968 relative à l'exercice du droit syndical dans l'entreprise», in Le Crom J.-P. (sous la direction de), Deux siècles de droit du travail, l'histoire par les lois, Paris, Édition de l'Atelier, pp. 213-228.

Crozier M. (1970), La société bloquée, Paris, Seuil.

Delors J. (1965), «Planification et réalités syndicales », Droit Social, n 3, mars, pp. 154-160.

Delors J. (2003), Mémoires, Paris, Plon.

Dupré M., Giraud O., Tallard M., Vincent C. (2006), «L'État et les acteurs sociaux face à la démocratie industrielle en France et en Allemagne entre 1945 et les années 1980 », in Chatriot A., Join-Lambert O., Viet V. (sous la direction de), Les politiques du travail (1906-2006). Acteurs, institutions, réseaux, Presses universitaires de Rennes, pp. 343-358.

Frerich J., Frey M. (1993), Handbuch der Geschichte der Sozialpolitik in Deutschland, Bd 3, Munich, Richard Oldenburg Verlag.

Frachon B. (1948), Revue des comités d'entreprise, $\mathrm{n}^{\circ} 1$, avril.

Georgi F. (1995), L'invention de la CFDT 1957-1970, Paris, Éditions de l'Atelier.

Guyot P. (2001), «La politique sociale du gaullisme», Éducation permanente $\mathrm{n}^{\circ} 149$, pp. 59-96.

Hall P.A. (1997), "The Political Economy of Adjustment in Germany", in Naschol F., Soskice D., Hanche B., Jürgens U. (sous la direction de), Ökonomische Leistungsfähigkeit und institutionnelle Innovation - Das deutsche Produktions - und Politikregime im globalen Wettbewerb, Berlin, Edition Sigma, pp. 293-317.

Hensche D. (1976), Grundsatzprogramm und Wirtschaftsordnung - Zur gewerkschaftlichen Programmatik seit 1945, Gewerkschaftliche Monatshefete, 11, pp. 688696.

HöPNER M. (2004), Sozialdemokratie, Gewerkschaften und organisierter Kapitalismus, 1880-2002, MPIfG Discussion Paper 2004/10. Köln, Max-Planck-Institut für Gesellschaftsforschung.
Jobert A. (2000), Les espaces de la négociation collective, branches et territoires, Toulouse, Octares éditions.

King C.D., Van De Vall (1978), Models of Industrial Democracy: Consultation, Co-Determination, and Workers' Management, La Haye, Mouton.

KlÖNE A, REESE H. (1990), "Zeiten des Umbruchs - Die Gewerkschaften unter der Großen Koalition", in Hemmer H.O., Schmitz K.T. (sous la direction de), Geschichte der Gewerkschaften in der Bundesrepublik Deutschland-Von den Anfängen bis heute, Cologne, Bund Verlag, pp. 251-279.

LE CROM J.-P.(2001), L'introuvable démocratie salariale, le droit de la représentation du personnel dans l'entreprise, Paris, Éditions Syllepse.

Lompa K. (1990), “Gewerkschaftliche Politik in der Phase gesellschaftlicher Reformen und der außenpolitischer Neuorientierung der Bundesrepublik 1969 bis 1974", in Hemmer H.O., Schmitz K.T. (sous la direction de), Geschichte der Gewerkschaften in der Bundesrepublik Deutschland - Von den Anfängen bis heute, Cologne, Bund Verlag, pp. 251-279.

Magnadas J. (1985), «Le CNPF dans la lutte des classes», Société française, $\mathrm{n}^{\circ} 17$, octobre-décembre, pp. 10-24.

Mathevet R. (1965), «Le syndicalisme dans l'entreprise, à propos de l'extension du droit syndical dans l'entreprise», rapport présenté au nom de la section des activités sociales du Conseil économique et social, Droit Social, $\mathrm{n}^{\circ}$ 3, mars, pp. 174-190.

Maurice M., Sellier F., Silvestre J.-J. (1982), Politique d'éducation et d'organisation industrielle en France et en Allemagne. Essai d'analyse sociétale, Paris, PUF.

Mouriaux R. (1982), La CGT, Paris, La Découverte.

Moynot J.-L. (1982), Аи milieu du gué, Paris, PUF.

NûtZnAdel A. (2005), Stunde der Ökonomen, Göttingen, Vandenhoeck \& Ruprecht.

OFFE K. (1975), Berufsbildungsreform - Eine Fallstudie über Reformpolitik, Francfort s.1.M. : Suhrkamp.

Reynaud J.-D. (1999), «Les évènements de mai-juin 1968 et le système français de relations professionnelles », in Le conflit, la négociation et la règle, Toulouse, Octarès éditions (première publication: Sociologie $d u$ travail $\mathrm{n}^{\circ} 1,1971$ pp. 73-97 et $\mathrm{n}^{\circ} 2$ pp. 121-209).

Rotschild-Souriac M.-A. (1993), «Autonomie de la négociation d'entreprise», in Jobert A., Reynaud J.-D., Saglio J., Tallard M. (sous la direction de), Les conventions collectives de branche: déclin ou renouveau? Paris, CEREQ.

Schroeder W. (2000), "Industrielle Beziehungen in den $60^{\text {er }}$ Jahren - unter besonderer Berücksichtigung 
der Metallindustrie", in Schildt A., Detlef S., Lammers K.C. (sous la direction de), Dynamische Zeiten-Die 60 er Jahre in den beiden deutschen Gesellschaften, Hamburg, Christians Verlag, pp. 492-527.

Sellier F.(1984), La confrontation sociale, 1936-1981, Paris, PUF.

Streeck W. (1985), "Industrial Relations in WestGermany 1974-1985: an Overview”, Berlin, WZBWorking Paper IIM/LMP 85-19.

Thelen K. (1991), Union of Parts - Labor Politics in Postwar Germany, Ithaca, Cornell University Press.

Verdier J.-M., Langlois P. (1972), «Aux confins de la théorie des sources du droit, une relation nouvelle entre la loi et l'accord collectif», Recueil Dalloz Sirey, 39e cahier, chronique.

VinCEnT C. (1997), «De l'accord de juillet 1970 à la loi de 1971 : l'échec d'un paritarisme négocié dans la formation professionnelle continue», La Revue de l'IRES, numéro spécial «Le paritarisme: institutions et acteurs », $\mathrm{n}^{\circ} 24$, pp. 153-173.

Webbs S., Webbs B. (1897), Industrial Democracy, Longmans, Green \& Co., deux volumes.

Zimmermann B., Didry C., Wagner P. (sous la direction de) (1999), Le travail et la nation. Histoire croisée de la France et de l'Allemagne, Paris, éditions de la Maison des sciences de l'homme. 